\title{
Dedifferentiated Adenoid Cystic Carcinoma: A Clinicopathologic Study of 6 Cases
}

Toshitaka Nagao, M.D., Thomas A. Gaffey, M.D., Hiromi Serizawa, M.D., Isamu Sugano, M.D., Yasuo Ishida, M.D., Kazuto Yamazaki, M.D., Ryoji Tokashiki, M.D., Tomoyuki Yoshida, M.D., Hiroshi Minato, M.D., Paul A. Kay, M.D., Jean E. Lewis, M.D.

Division of Anatomic Pathology, Mayo Clinic, Rochester, Minnesota (TN, TAG, HM, PAK, JEL); the Departments of Surgical Pathology (HS) and Otolaryngology (RT, TY), Tokyo Medical University Hospital, Tokyo, Japan; and the Department of Surgical Pathology (IS, YI, KY), Teikyo University Ichihara Hospital, Teikyo, Japan.

Dedifferentiated adenoid cystic carcinomas are a recently defined, rare variant of adenoid cystic carcinomas characterized histologically by two components: conventional low-grade adenoid cystic carcinoma and high-grade "dedifferentiated" carcinoma. We examined six cases and analyzed their clinicopathologic profiles, including immunohistochemical features and p53 gene alterations. The 6 patients $(3$ men and 3 women) had a mean age of 46.8 years (range, 34-70 y). The mean size of the tumors was $3.5 \mathrm{~cm}$ (range, 1.7-6 cm). The submandibular gland, maxillary sinus, and nasal cavity were involved in 2 cases each. Postoperatively, 5 patients had local recurrence and 5 developed metastatic disease. Five patients died of disease at a mean of 33.7 months after diagnosis (range, 6-69 mo), and one other was alive with disease at 60 months. Histologically, the conventional low-grade adenoid cystic carcinoma component of the tumors consisted of a mixture of cribriform and tubular patterns with scant solid areas. The high-grade dedifferentiated carcinoma component was either a poorly differentiated adenocarcinoma ( 4 cases) or undifferentiated carcinoma ( 2 cases). Three tumors were studied immunohistochemically. Myoepithelial markers were expressed in low-grade adenoid cystic carcinoma but not in the dedifferentiated component. In 2 cases, diffusely positive p53 immuno-

Copyright (C) 2003 by The United States and Canadian Academy of Pathology, Inc.

VOL. 16, NO. 12, P. 1265, 2003 Printed in the U.S.A.

Date of acceptance: August 15, 2003.

TN and HM are Visiting Clinicians in the Division of Anatomic Pathology. Copyright 2003 Mayo Foundation.

Address reprint requests to: Thomas A. Gaffey, M.D., Division of Anatomic Pathology, Mayo Clinic, 200 First Street SW, Rochester, MN 55905; fax: 507-284-1599; e-mail: gaffey.thomas@mayo.edu.

DOI: $10.1097 / 01 . M P .0000097366 .88165 .08$ reactivity together with HER-2/neu overexpression was restricted to the dedifferentiated component. Loss of pRb expression was demonstrated only in the dedifferentiated component of the 1 other case. The Ki-67-labeling index was higher in the dedifferentiated component than in the lowgrade adenoid cystic carcinoma component. Furthermore, molecular analysis of 2 cases demonstrated the loss of heterozygosity at $p 53$ microsatellite loci, accompanied by $p 53$ gene point mutation, only in the dedifferentiated carcinoma component of 1 case, which was positive for p53 immunostaining. These results indicate that dedifferentiated adenoid cystic carcinoma is a highly aggressive tumor. Because of frequent recurrence and metastasis, the clinical course is short, similar to that of adenoid cystic carcinomas with a predominant solid growth pattern. Limited evidence suggests that $p 53$ abnormalities in combination with HER-2/neu overexpression or loss of $\mathbf{p R b}$ expression may have a role in dedifferentiation of adenoid cystic carcinoma.

KEY WORDS: Adenoid cyst, Carcinoma, Dedifferentiation, HER-2/neu, p53, Rb, Salivary gland.

Mod Pathol 2003;16(12):1265-1272

Adenoid cystic carcinomas are common malignancies arising in major and minor salivary glands, including seromucinous glands of the upper respiratory tract. They are characterized clinically by an indolent course, a relatively high rate of local recurrence, and late onset of distant metastases. Histologically, adenoid cystic carcinomas may have a mixture of 3 distinctive growth patterns: cribriform, tubular, and solid. The most common histologic type is the cribriform pattern, in which monomorphic tumor cells form islands with punched-out spaces, producing a so-called Swiss-cheese appearance. The predominant growth pattern is predictive 
to some extent of clinical outcome. Most studies have suggested that adenoid cystic carcinomas with both tubular and cribriform patterns generally have a better prognosis than those with the solid pattern (1-4).

In 1971, Dahlin and Beabout (5) introduced the term "dedifferentiated chondrosarcoma" to describe a distinct clinicopathologic entity in which a low-grade chondrosarcoma was associated withbut sharply delineated from-a histologically dissimilar high-grade sarcoma. These cases were characterized by aggressive clinical behavior. Recent studies have reported that some low-grade salivary gland carcinomas such as acinic cell carcinoma (6-11), adenoid cystic carcinoma (12-14), epithelial-myoepithelial carcinoma $(15,16)$, and polymorphous low-grade adenocarcinoma $(17,18)$ undergo "dedifferentiation," although the frequency may be low. In such circumstances, the dedifferentiated element, which may be either undifferentiated carcinoma or poorly differentiated adenocarcinoma, lacks the original distinctive growth pattern. Dedifferentiation in low-grade adenoid cystic carcinoma, a recently reported phenomenon, is extremely rare (12). Therefore, the biologic behavior, immunohistologic, and molecular characteristics of dedifferentiated adenoid cystic carcinomas are not well understood.

In the present study, we documented the clinicopathologic features of 6 cases of dedifferentiated adenoid cystic carcinoma. Also, in selected cases, immunohistochemical and p53 gene alteration analyses were performed to clarify the molecular and genetic changes involved in the pathway of dedifferentiation.

\section{MATERIALS AND METHODS}

Six cases of dedifferentiated adenoid cystic carcinoma were identified from the pathology files of
Tokyo Medical University Hospital (Case 1), Teikyo University Ichihara Hospital (Case 2), Mayo Clinic (Cases 3 and 4), and the consultation files of 1 of the authors (JEL, Cases 5 and 6). The 6 cases fulfilled the criteria for dedifferentiated adenoid cystic carcinoma defined by Cheuk et al. (12), and cases of the solid type of adenoid cystic carcinoma were excluded from this study. Tumor tissue was fixed in $10 \%$ buffered formalin, processed by routine histologic techniques, and stained with periodic acidSchiff (periodic acid-Schiff) and Alcian blue. Clinical details and follow-up data were obtained when possible from hospital records, referring pathologists, and treating physicians.

\section{Immunohistochemistry}

For three of the six cases, paraffin blocks were available for immunohistochemical analysis. The deparaffinized and rehydrated slides were boiled in 10-mm citrate buffer, $\mathrm{pH} 6.0$, for 20 minutes in a microwave oven. After cooling for 30 minutes, tissue sections were incubated with the primary antibody for 15 hours at $4^{\circ} \mathrm{C}$. The primary antibodies used in this study are listed in Table 1. The labeled streptavidin-biotin peroxidase method (LSAB Kit DAKO, Carpinteria, CA) was used for detection, employing 3,3'-diaminobenzidine as the chromogen. The sections were lightly counterstained with hematoxylin. The percentage of Ki-67-positive cells was determined by counting at least 1000 tumor cells, and the value was recorded as the Ki-67labeling index (LI).

\section{Molecular Analysis}

DNA was available from two cases (Cases 1 and 2). With microdissection methods, DNA was extracted carefully in both cases from the paraffin-embedded sections of the two histologically different tumor

TABLE 1. Antibodies Used and Results of Immunohistochemistry

\begin{tabular}{|c|c|c|c|c|c|c|c|c|c|}
\hline \multirow{3}{*}{ Antigen (antibody) } & \multirow{3}{*}{ Source } & \multirow{3}{*}{ Clonality } & \multirow{3}{*}{ Dilution } & \multicolumn{6}{|c|}{ Results } \\
\hline & & & & \multicolumn{2}{|c|}{ Case 1} & \multicolumn{2}{|c|}{ Case 2} & \multicolumn{2}{|c|}{ Case 3} \\
\hline & & & & ACC & DC & ACC & $\mathrm{DC}$ & ACC & DC \\
\hline Cytokeratin (AE1/AE3) & DAKO, Carpinteria, CA & M & $1: 50$ & + & + & + & + & + & + \\
\hline EMA & DAKO & M & $1: 100$ & + & + & + & + & + & + \\
\hline$\alpha$-Smooth muscle actin & DAKO & M & $1: 150$ & $\mathrm{~F}+$ & - & + & - & + & - \\
\hline Muscle-specific actin & DAKO & M & $1: 50$ & + & - & + & - & + & - \\
\hline S-100 protein & DAKO & $\mathrm{P}$ & $1: 500$ & + & $\mathrm{F}+$ & + & + & + & $\mathrm{F}+$ \\
\hline GFAP & DAKO & M & $1: 100$ & - & - & - & - & - & - \\
\hline CEA & DAKO & M & $1: 50$ & + & + & $\mathrm{F}+$ & $\mathrm{F}+$ & $\mathrm{F}+$ & $\mathrm{F}+$ \\
\hline HER-2/neu & Novocastra Lab., Newcastle, UK & M & $1: 40$ & - & + & - & - & - & + \\
\hline p53 (DO7) & Novocastra Lab. & M & $1: 100$ & - & + & - & - & $\mathrm{F}+$ & + \\
\hline $\mathrm{pRb}$ & Pharmingen, San Diego, CA & M & $1: 100$ & + & + & + & - & + & + \\
\hline Cyclin D1 & Novocastra Lab. & M & $1: 50$ & $\mathrm{~F}+$ & - & - & $\mathrm{F}+$ & - & $\mathrm{F}+$ \\
\hline Ki-67 (MIB-1) (\%) & Immunotech S.A., Marseille, France & M & $1: 200$ & 11.2 & 56.1 & 5.2 & 42.3 & 4.7 & 45.1 \\
\hline
\end{tabular}

ACC, low-grade adenoid cystic carcinoma component; CEA, carcinoembryonic antigen; DC, dedifferentiated carcinoma component; EMA, epithelial membrane antigen; F, focal; GFAP, glial fibrillary acidic protein; M, monoclonal; P, polyclonal. 
components (i.e., conventional low-grade adenoid cystic carcinoma and dedifferentiated carcinoma) and adjacent normal tissue. The primer sequences for polymerase chain reaction (PCR) amplification were the same as those described previously (19). Forward primers of TP53 locus and a variable tandem repeats (VNTR) section in p53 intron 1 were labeled with 6-FAM for loss-of-heterozygosity ( $\mathrm{LOH}$ ) analysis. $\mathrm{LOH}$ analysis was performed with a fluorescence-based microsatellite PCR technique using ABI PRISM 310 Genetic Analyzer (PerkinElmer, Foster City, CA) and Gene Scan 2.01 software (PerkinElmer). The ratios of the normal and tumor allele peak areas were compared for $\mathrm{LOH}$ assessment. A change in the ratio by $>50 \%$ indicated the loss of one allele in the carcinoma DNA (20). Mutational analysis was performed for $p 53$ exon 5-8 by direct DNA sequencing. The DNA was sequenced with dRhodamine Terminator Cycle Sequencing Ready Reaction Kit (PerkinElmer) and $\mathrm{ABI}$ PRISM 310 Genetic Analyzer (PerkinElmer).

\section{RESULTS}

The clinicopathologic features of dedifferentiated adenoid cystic carcinoma are summarized in Table 2 . The 6 patients ( 3 men and 3 women) had a mean age of 46.8 years (range, 34-70 y). The mean size of the tumors was $3.5 \mathrm{~cm}$ (range, $1.7-6 \mathrm{~cm}$ ). Tumor size was not available for one case. In two cases each, the tumor involved the submandibular gland, maxillary sinus, and nasal cavity. The patients underwent excision of the tumor or total glandectomy, with or without postoperative chemotherapy or radiotherapy. Postoperatively, five patients had local recurrence of the tumor and 5 developed metastatic disease. Metastatic lesions occurred in bone (3 cases), lungs (2 cases), and cervical lymph nodes (3 cases). Five patients died of disease at a mean of 33.7 months (range, 6-69 mo) after the initial diagnosis. The one other patient was alive with disease at 60 months.

Histologically, the six neoplasms were composed of two distinct carcinomatous components. One component was a conventional low-grade adenoid cystic carcinoma, and the other was a high-grade dedifferentiated carcinoma (Fig. 1). The dedifferentiated component was the predominant feature in all cases. Generally, both the conventional lowgrade adenoid cystic carcinoma and high-grade dedifferentiated carcinoma components were clearly separated from each other. The low-grade neoplasm consisted of a mixture of cribriform and tubular patterns. However, the proportion of lowgrade growth patterns varied from case to case. The cribriform pattern was predominant in three cases and the tubular pattern, in the other three. The cribriform pattern had cyst-like spaces (pseudo-

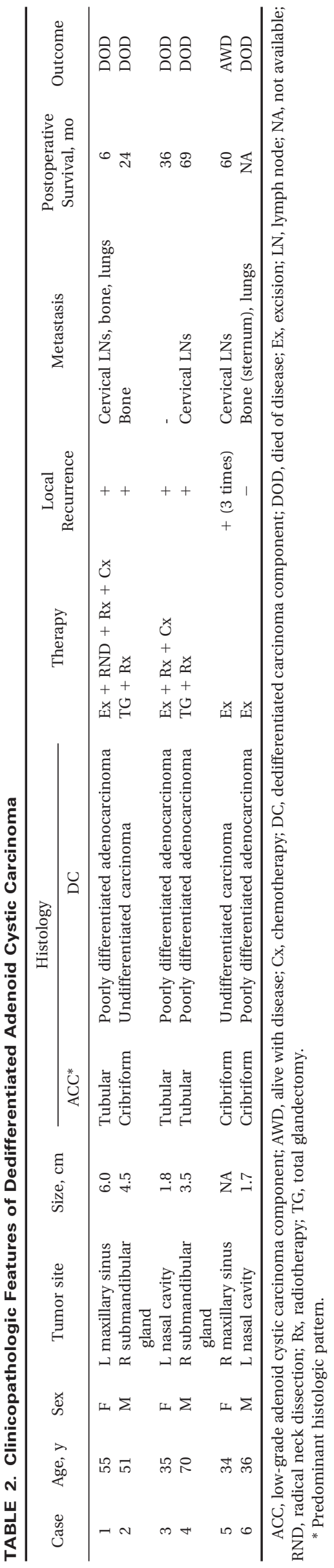




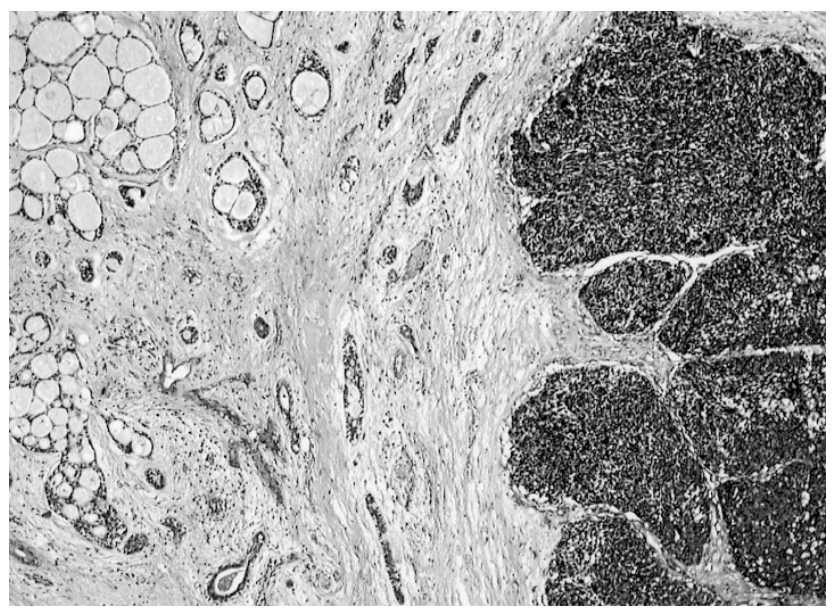

FIGURE 1. Low-power view showing, on the left, low-grade adenoid cystic carcinoma and, on the right, dedifferentiated carcinoma with solid cell nests. The two components are sharply separated from each other.

cysts) containing periodic acid-Schiff- and Alcian blue-positive, pale basophilic or eosinophilic basal lamina material (Fig. 2A). Scattered small, true

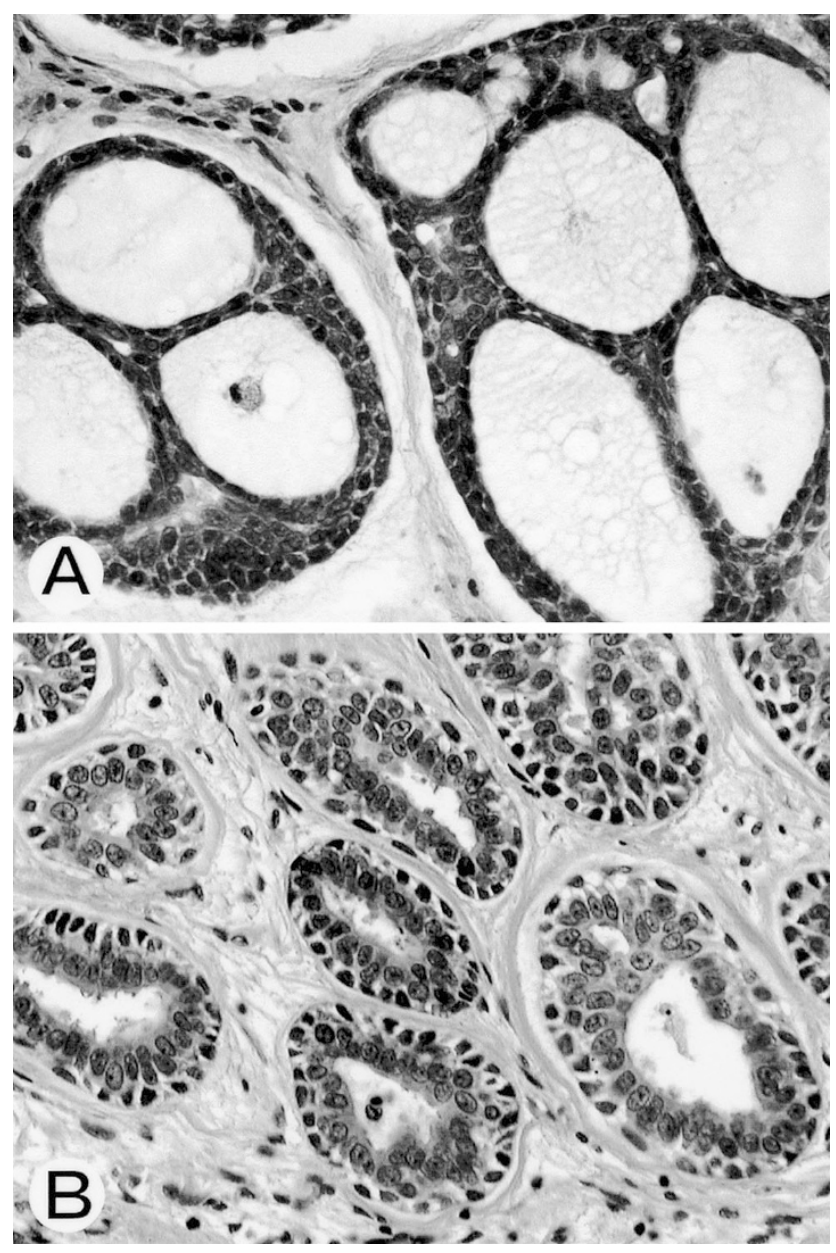

FIGURE 2. Low-grade adenoid cystic carcinoma component. The tumor cell nuclei have a bland, uniform appearance. A, cribriform pattern. B, tubular structures with bicellular layers. glandular structures were also observed. Tubular structures had an inner layer of duct-lining cells and an outer layer of clear cells (Fig. 2B). Alcian blue-positive mucoid material occasionally surrounded the tubular cell nests. Solid cell nests were scanty in all cases of conventional adenoid cystic carcinoma.

Two cell types were identified in the low-grade adenoid cystic carcinoma of all cases. The more frequent cell type had small, angular dark nucleus with scant cytoplasm, and the other type was a duct-like cuboidal cell. Nuclei in the low-grade component had a bland, relatively uniform appearance without pleomorphism. Mitotic figures were rare. Perineural invasion was easily identified. In comparison, the dedifferentiated, high-grade carcinoma was either poorly differentiated adenocarcinoma (Cases 1, 3, 4, and 6; Fig. 3A-B) or undifferentiated carcinoma (Cases 2 and 5; Fig. 3C -D). Poorly differentiated adenocarcinoma usually had a predominantly solid growth pattern, forming irregular tumor nests with a few tubular structures (Fig. 3A). Papillary features were present focally in Case 1 (Fig. 3A). A trabecular pattern within the fibrous stroma was also seen in Cases 3 and 6 (Fig. 3B). Undifferentiated carcinoma was characterized by solid cell nests with streaming and scattered small squamous eddy-like whorls (Fig. 3C-D). Necrosis was commonly seen, and central comedolike necrosis superficially resembling salivary duct carcinoma was identified in Cases 1 and 6 (Fig. 3A).

In all cases, the high-grade carcinoma cells exhibited large pleomorphic nuclei with a moderate amount of cytoplasm and high mitotic activity. These tumor nuclei had vesicular chromatin with conspicuous nucleoli (Fig. 3). The cytomorphologic features of high-grade carcinoma cells were different from those of the cells observed in the solid type

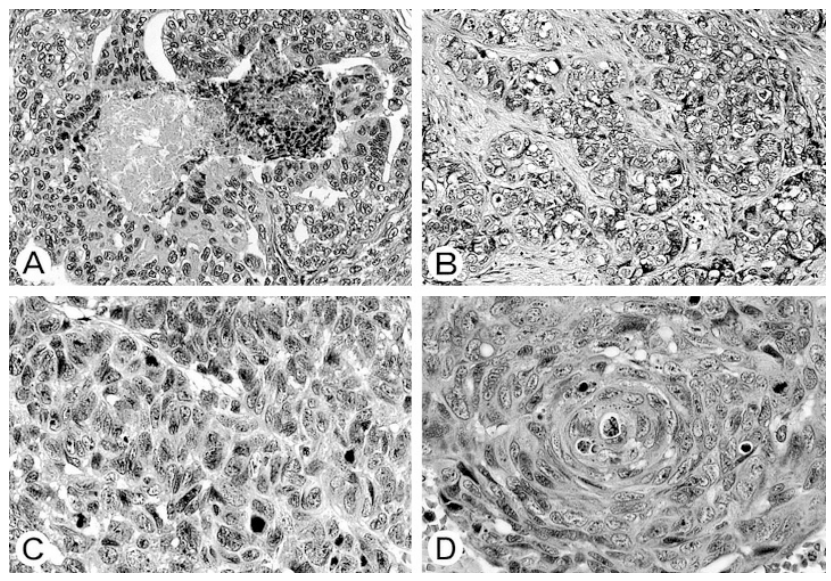

FIGURE 3. Dedifferentiated carcinoma component. A-B, poorly differentiated adenocarcinoma. C-D, undifferentiated carcinoma. Note tumor cell pleomorphism. A, papillary and tubular growth patterns with comedo-like necrosis. B, trabecular pattern with fibrous stroma. C, solid pattern of growth. D, squamous eddy-like whorl formation. 
of adenoid cystic carcinoma; the latter cells usually have small, densely hyperchromatic, monotonousappearing nuclei, and scant cytoplasm. Lymphovascular invasion was observed frequently. Alcian blue-positive mucinous material was absent in the dedifferentiated areas. Although low-grade adenoid cystic carcinoma and dedifferentiated carcinoma components usually were distinct (Fig. 1), in some cases a transitional zone was recognized between them (Fig. 4). The metastatic lesions available for review contained only the high-grade component and involved a cervical lymph node in Case 1 and bone in Case 6.

The results of immunohistochemical staining of three cases of dedifferentiated adenoid cystic carcinoma are presented in Table 1. Immunohistochemically, the cells located mainly on the inner (luminal) aspect of cell nests of low-grade adenoid cystic carcinomas stained positively for both cytokeratins (AE1/AE3) and epithelial membrane antigen. Conversely, $\alpha$-smooth muscle actin and muscle-specific actin, indicators of myoepithelial cell differentiation, were localized to abluminal tumor cells adjacent to the connective tissue. In comparison, dedifferentiated carcinoma cells expressed diffuse immunoreactivity for cytokeratins (AE1/ AE3) and epithelial membrane antigen but not for $\alpha$-smooth muscle actin or muscle-specific actin, indicating loss of myoepithelial cell differentiation. Both low-grade adenoid cystic carcinomas and the dedifferentiated neoplasms showed S-100 protein immunostaining in all cases, but only focally in dedifferentiated components of Cases 1 and 3. Immunostaining for glial fibrillary acidic protein was negative throughout the tissue in all cases.

In all cases, both components showed positive reaction for carcinoembryonic antigen, but carcinoembryonic antigen-positive cells were distributed focally in Cases 2 and 3. Strong membranous

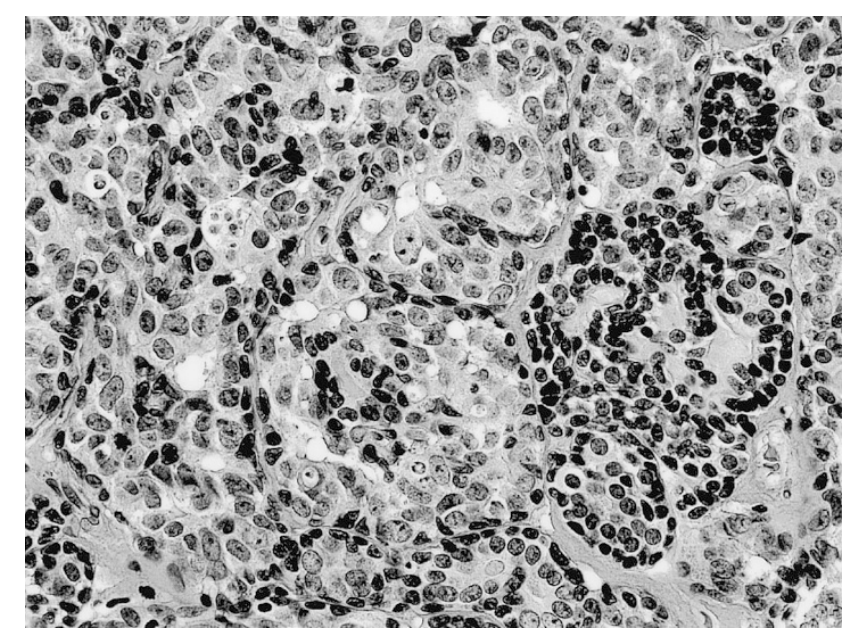

FIGURE 4. Transitional zone between low-grade adenoid cystic carcinoma and dedifferentiated carcinoma. immunoreactivity for HER-2/ neu was detected only in dedifferentiated components of Cases 1 and 3 (Fig. 5A). Most of the dedifferentiated carcinoma cells in Cases 1 and 3 showed strong nuclear p53 immunostaining (Fig. 5B). In low-grade adenoid cystic carcinoma, focal, and weak positivity for p53 was found in Case 3, but Case 1 was completely negative (Fig. 5B). No immunoreactivity for p53 was detected in Case 2, and loss of pRB expression was demonstrated only in the dedifferentiated component of this case (Fig. 5C-D). Cyclin D1 was expressed focally only in the low-grade adenoid cystic carcinoma component of Case 1 and in the dedifferentiated component of Cases 2 and 3. The Ki-67 LI was consistently higher in dedifferentiated carcinoma than in low-grade adenoid cystic carcinoma. The Ki-67 LI of the dedifferentiated carcinoma and the low-grade adenoid cystic carcinoma was $56.1 \%$ and $11.2 \%$, respectively, in Case $1,42.3 \%$ and $5.2 \%$ in Case 2 (Fig. 5E-F), and $45.1 \%$ and $4.7 \%$ in Case 3.

DNA was available for molecular analysis for Cases 1 and 2. Gene alteration analysis of p53 revealed that only Case 1 showed LOH at TP53 (Fig. 6A) accompanied by point mutation: TTT to ATT transition in codon 270 on exon 8, resulting in a Phe

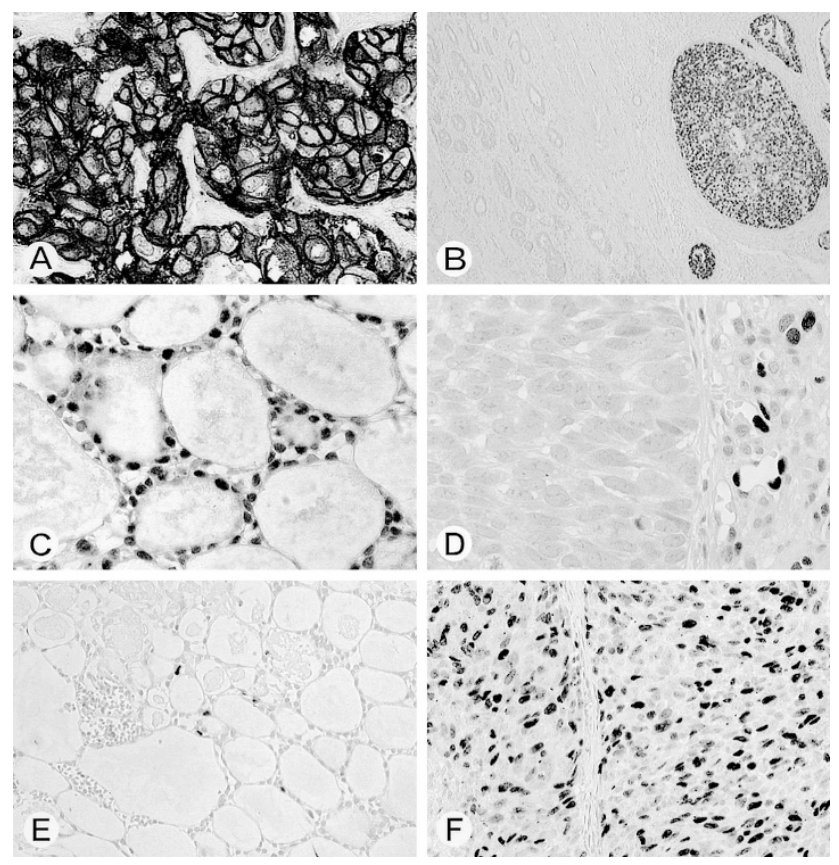

FIGURE 5. Immunohistochemistry of dedifferentiated adenoid cystic carcinoma. A, strong membranous positivity for HER-2/neu in dedifferentiated carcinoma cells. B, the dedifferentiated carcinoma component on the right is strongly positive for p53, whereas the conventional low-grade adenoid cystic carcinoma component on the left is completely negative. C-D, the majority of low-grade adenoid cystic carcinoma cells (C) show positive nuclear immunoreactivity for pRb, but its expression is lost in dedifferentiated cells (D). Note positive staining in endothelial cells as internal control (D). E-F, apparently different Ki-67 (MIB-1) immunoreactivity can be noted between the low-grade adenoid cystic carcinoma (labeling index, 5.2\%; E) and the dedifferentiated carcinoma components (labeling index, 42.3\%; F). 
NT

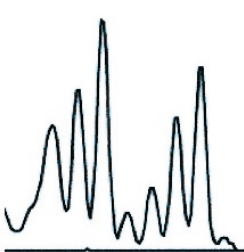

ACC

DC

A

\section{ACC \\ DC}

AC AG C $\underline{T T T}$ G AG G

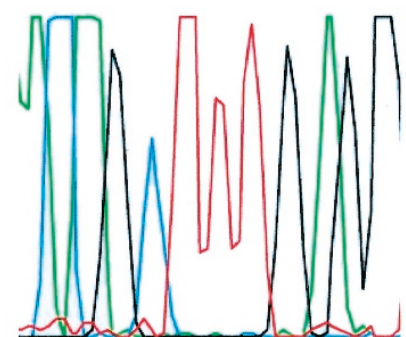

B Mutation (-)

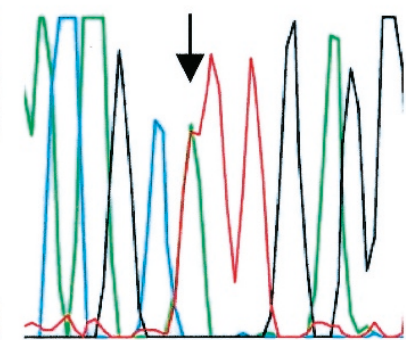

Mutation (+)
FIGURE 6. p53 gene alteration analysis. A, fluorescent loss of heterozygosity analysis. Longer allele showing marked signal reduction only in dedifferentiated carcinoma component (arrow). B, direct sequencing analysis of $p 53$ exon 8 . Dedifferentiated carcinoma component showing TTT to ATT transition (arrow) in codon 270 results in a Phe substitution for Ile, whereas no mutations are found in adenoid cystic carcinoma (low-grade) component. Vertical axes, fluorescent signal; horizontal axes, DNA size. ACC, adenoid cystic carcinoma (low-grade); DC, dedifferentiated carcinoma; NT, nontumor.

substitution for Ile (Fig. 6B), which was restricted to the dedifferentiated carcinoma component. VNTR in p53 intron 1 was not informative (homozygous) in either Case 1 or 2.

\section{DISCUSSION}

"Dedifferentiation" is defined as the abrupt transformation of a low-grade, well-differentiated tumor into a tumor of high-grade morphology. It is a well-recognized phenomenon in bone and soft tissue tumor pathology (21). Dedifferentiated tumors generally are associated with a poor prognosis. After Stanley et al. (6) first reported the dedifferentiated variant of salivary gland acinic cell carcinoma in 1988, several investigators have described it not only in acinic cell carcinoma (7-11) but also in other low-grade salivary carcinomas (12-18). Although the concept of dedifferentiation appears to be established for salivary gland neoplasms (22), this phenomenon is uncommon in adenoid cystic carcinoma. To our knowledge, only five cases have been reported (Table 3) (12-14). Altogether, from our six cases and the previously reported five cases (12-14), dedifferentiated adenoid cystic carcinoma has affected five men and six women, who had a mean age of 50.1 years (range, 34-70 y). The tumors preferentially developed in intraoral minor salivary glands and seromucinous glands of the upper respiratory tract. Dedifferentiation may be present when an untreated (primary) low-grade salivary gland tumor is detected or it can develop in a recurrent, previously treated, initially low-grade tumor $(5,22)$. Although two previously reported cases of dedifferentiation in adenoid cystic carcinoma developed in recurrent tumor sites after radiotherapy (12), all our cases were diagnosed ab initio. Therefore, the possible role of radiotherapy as an initiator of dedifferentiation in adenoid cystic carcinoma is an unsettled issue. Of nine patients with long-term follow-up, six died of disease within 3 years after the initial diagnosis, accompanied by recurrence and metastasis. These data indicate that dedifferentiated adenoid cystic carcinoma is a highly aggressive tumor with a strong tendency to recur and metastasize, similar to that observed in adenoid cystic carcinomas with a predominantly solid growth pattern $(1-4,23)$. Increased cell proliferative activity, assessed with Ki-67 (MIB-1) immunostaining, in the dedifferentiated components of our cases also correlates with the high-grade malignant nature of this tumor (24).

TABLE 3. Cases of Dedifferentiated Adenoid Cystic Carcinoma Reported in the Literature

\begin{tabular}{|c|c|c|c|c|c|c|c|c|c|}
\hline Case & Reference & Year & Age, y & Sex & Tumor Site & Size, $\mathrm{cm}$ & Histology of DC & Metastasis & Follow-up \\
\hline 1 & Cheuk et al. (12) & 1999 & 55 & $\mathrm{~F}$ & Tongue & NA & $\begin{array}{l}\text { Poorly differentiated } \\
\text { adenocarcinoma }\end{array}$ & $\begin{array}{r}\text { Cervical LNs, } \\
\text { bone, lung }\end{array}$ & $\mathrm{DOD}$ at $15 \mathrm{mo}$ \\
\hline 2 & Cheuk et al. (12) & 1999 & 53 & $\mathrm{~F}$ & Soft palate & NA & $\begin{array}{l}\text { Sarcomatoid } \\
\text { carcinoma with } \\
\text { myoepithelial } \\
\text { differentiation }\end{array}$ & Lung & DOD at $9 \mathrm{mo}^{*}$ \\
\hline 3 & Cheuk et al. (12) & 1999 & 38 & M & Hard palate & NA & $\begin{array}{l}\text { Poorly differentiated } \\
\text { adenocarcinoma }\end{array}$ & Cervical LNs & DOD at $18 \mathrm{mo}^{*}$ \\
\hline 4 & Moles et al. (13) & 1999 & 61 & M & Tongue & 3 & $\begin{array}{l}\text { Undifferentiated } \\
\text { carcinoma, large } \\
\text { cell type }\end{array}$ & - & $\mathrm{NED}$ at $60 \mathrm{mo}$ \\
\hline 5 & Chau et al. (14) & 2001 & 64 & $\mathrm{~F}$ & $\begin{array}{l}\text { L submandibular } \\
\text { gland }\end{array}$ & 3 & $\begin{array}{c}\text { Poorly differentiated } \\
\text { adenocarcinoma }\end{array}$ & Cervical LNs & $\mathrm{NED}$ at $6 \mathrm{mo}$ \\
\hline
\end{tabular}

DC, dedifferentiated carcinoma component; DOD, died of disease; LN, lymph node; NA, not available; NED, no evidence of disease.

* Dedifferentiation occurred in the recurrence of conventional low-grade adenoid cystic carcinoma. 
In practice, the potential for dedifferentiation in adenoid cystic carcinoma warrants thorough sampling of all initially diagnosed adenoid cystic carcinomas (22).

Before the diagnosis of dedifferentiated adenoid cystic carcinoma is made, other types of salivary gland neoplasms with morphologically high-grade features that mimic low-grade adenoid cystic carcinoma should be excluded. These include the solid type of adenoid cystic carcinoma $(1-4,23)$, collision tumors, and hybrid carcinomas $(25,26)$. Even in the solid type, the tumor cells of adenoid cystic carcinoma exhibit the original line of differentiation. However, there may be a histologic spectrum between some cases of the solid type of adenoid cystic carcinoma and the dedifferentiated component of our cases. Similar to the dedifferentiated component, cellular atypia, occasional comedolike necrosis, and frequent mitotic figures may be seen in the solid type of adenoid cystic carcinoma. However, in the solid type of adenoid cystic carcinoma, solid cell nests are commonly intermixed with cribriform and tubular structures throughout the tumor, instead of being clearly separated from them as in dedifferentiated adenoid cystic carcinoma. Also, the cytologic details help to distinguish the two entities. The cells of the solid type of adenoid cystic carcinoma have a basaloid appearance characterized by small, hyperchromatic nuclei with scant cytoplasm. In contrast, dedifferentiated carcinoma cells have larger, more pleomorphic, and vesicular nuclei with a moderate nucleocytoplasmic ratio. Furthermore, the solid type of adenoid cystic carcinoma may retain focal myoepithelial differentiation. Dedifferentiated carcinoma, in our cases, was completely negative for both $\alpha$-smooth muscle actin and muscle-specific actin.

The presence of a transitional zone between the two carcinoma components, suggesting an identical origin, distinguishes our tumors from true collision tumors, which are a union of two separate neoplasms arising from independent topographic sites. Hybrid carcinomas, composed of two forms of malignancy that fit into precisely defined tumor categories, produce a single tumor mass within the same topographic area $(25,26)$. Although hybrid carcinoma consisting of adenoid cystic carcinoma and salivary duct carcinoma may be included in the differential diagnosis of dedifferentiated adenoid cystic carcinoma, the dedifferentiated component in our cases did not exhibit certain features specific for salivary duct carcinoma, for example, proliferation of pleomorphic carcinoma cells with eosinophilic cytoplasm and prominent nucleoli, cribriform (so-called Roman bridge) formation, comedo necrosis, and often desmoplastic reaction (27). Two cases of hybrid carcinoma composed of adenoid cystic carcinoma and salivary duct carcinoma have been reported, but from our review of the literature, they possibly are examples of dedifferentiated adenoid cystic carcinoma $(28,29)$.

The development and progression of cancer are thought to be regulated by the expression of various oncogenes and tumor suppressor genes. Their activity has been documented in salivary gland tumors $(19,26,30-33)$. In conventional adenoid cystic carcinoma, $\mathrm{LOH}$ at either the $p 53$ or $R B$ gene was detected more frequently in the solid portions than in the cribriform and tubular areas within the same tumor mass (32). Thus, progression from the cribriform and tubular types of adenoid cystic carcinoma to the solid type has been postulated. Also, p53 gene mutation has been implicated in dedifferentiation of chondrosarcoma (34) but not of acinic cell carcinoma $(9,10)$. To elucidate the mechanism of dedifferentiation in adenoid cystic carcinoma, we examined the expression of tumor suppressor gene products (p53 and pRb), oncogene product (HER-2/neu), and cyclin D1, an important regulator of the G1 phase of the cell cycle. Furthermore, $p 53$ gene alteration analysis was performed on selected cases. In two of three cases, simultaneous aberrant expression of p53 and HER-2/ neu was restricted to the dedifferentiated component and was not found in low-grade adenoid cystic carcinoma. Coordinate overexpression of p53 and p53 gene alteration was observed in one of these "restricted" cases. Previous studies also have reported p53 abnormalities in two of four examined cases $(12,14)$. Generally, coexpression of p53 and HER-2/ neu in salivary gland tumors is rare $(31,33)$ and has been found only in high-grade neoplasms such as adenocarcinoma, carcinoma ex pleomorphic adenoma, and salivary duct carcinoma (31). Loss of $\mathrm{pRb}$ expression occurred in only a single dedifferentiated carcinoma, which was negative for p53 and HER-2/ neu.

Although cyclin D1 overexpression may have a role in the dedifferentiation process of adenoid cystic carcinoma $(12,14)$, our study did not confirm its importance. Our results suggest that $p 53$ abnormalities in combination with HER-2/neu overexpression or loss of $\mathrm{pRb}$ expression may be important in dedifferentiation in low-grade adenoid cystic carcinoma.

\section{REFERENCES}

1. Perzin KH, Gullane P, Clairmont AC. Adenoid cystic carcinomas arising in salivary glands: a correlation of histologic features and clinical course. Cancer 1978;42:265-82.

2. Szanto PA, Luna MA, Tortoledo ME, White RA. Histologic grading of adenoid cystic carcinoma of the salivary glands. Cancer 1984;54:1062-9.

3. Nascimento AG, Amaral AL, Prado LA, Kligerman J, Silveira TR. Adenoid cystic carcinoma of salivary glands: a study of 61 cases with clinicopathologic correlation. Cancer 1986;57: 312-9. 
4. Batsakis JG, Luna MA, el-Naggar A. Histopathologic grading of salivary gland neoplasms: III. Adenoid cystic carcinomas. Ann Otol Rhinol Laryngol 1990;99:1007-9.

5. Dahlin DC, Beabout JW. Dedifferentiation of low-grade chondrosarcomas. Cancer 1971;28:461-6.

6. Stanley RJ, Weiland LH, Olsen KD, Pearson BW. Dedifferentiated acinic cell (acinous) carcinoma of the parotid gland. Otolaryngol Head Neck Surg 1988;98:155-61.

7. el-Naggar AK, Batsakis JG, Luna MA, McLemore D, Byers RM. DNA flow cytometry of acinic cell carcinomas of major salivary glands. J Laryngol Otol 1990;104:410-6.

8. Nunes JF, Fonseca I, Soares J. Helioid inclusions in dedifferentiated acinic cell carcinoma of the parotid gland. Ultrastruct Pathol 1996;20:443-9.

9. Henley JD, Geary WA, Jackson CL, Wu CD, Gnepp DR. Dedifferentiated acinic cell carcinoma of the parotid gland: a distinct rarely described entity. Hum Pathol 1997;28:869-73.

10. Di Palma S, Corletto V, Lavarino C, Birindelli S, Pilotti S. Unilateral aneuploid dedifferentiated acinic cell carcinoma associated with bilateral-low grade diploid acinic cell carcinoma of the parotid gland. Virchows Arch 1999;434:361-5.

11. Piana S, Cavazza A, Pedroni C, Scotti R, Serra L, Gardini G. Dedifferentiated acinic cell carcinoma of the parotid gland with myoepithelial features. Arch Pathol Lab Med 2002;126:1104-5.

12. Cheuk W, Chan JK, Ngan RK. Dedifferentiation in adenoid cystic carcinoma of salivary gland: an uncommon complication associated with an accelerated clinical course. Am J Surg Pathol 1999;23:465-72.

13. Moles MA, Avila IR, Archilla AR. Dedifferentiation occurring in adenoid cystic carcinoma of the tongue. Oral Surg Oral Med Oral Pathol Oral Radiol Endod 1999;88:177-80.

14. Chau Y, Hongyo T, Aozasa K, Chan JK. Dedifferentiation of adenoid cystic carcinoma: report of a case implicating p53 gene mutation. Hum Pathol 2001;32:1403-7.

15. Alos L, Carrillo R, Ramos J, Baez JM, Mallofre C, Fernandez $\mathrm{PL}$, et al. High-grade carcinoma component in epithelialmyoepithelial carcinoma of salivary glands: clinicopathological, immunohistochemical and flow-cytometric study of three cases. Virchows Arch 1999;434:291-9.

16. Fonseca I, Felix A, Soares J. Dedifferentiation in salivary gland carcinomas. Am J Surg Pathol 2000;24:469-71.

17. Pelkey TJ, Mills SE. Histologic transformation of polymorphous low-grade adenocarcinoma of salivary gland. Am J Clin Pathol 1999;111:785-91.

18. Simpson RH, Pereira EM, Ribeiro AC, Abdulkadir A, ReisFilho JS. Polymorphous low-grade adenocarcinoma of the salivary glands with transformation to high-grade carcinoma. Histopathology 2002;41:250-9.

19. Nagao T, Sugano I, Ishida Y, Tajima Y, Munakata S, Asoh A, et al. Primary large-cell neuroendocrine carcinoma of the parotid gland: immunohistochemical and molecular analysis of two cases. Mod Pathol 2000;13:554-61.

20. Cawkwell L, Bell SM, Lewis FA, Dixon MF, Taylor GR, Quirke P. Rapid detection of allele loss in colorectal tumours using microsatellites and fluorescent DNA technology. Br J Cancer 1993;67:1262-7.

21. Meis JM. "Dedifferentiation" in bone and soft-tissue tumors: a histological indicator of tumor progression. Pathol Annu 1991;26:37-62.

22. Zarbo RJ. Salivary gland neoplasia: a review for the practicing pathologist. Mod Pathol 2002;15:298-323.

23. Cleveland D, Abrams AM, Melrose RJ, Handlers JP. Solid adenoid cystic carcinoma of the maxilla. Oral Surg Oral Med Oral Pathol 1990;69:470-8.

24. Nordgard S, Franzen G, Boysen M, Halvorsen TB. Ki-67 as a prognostic marker in adenoid cystic carcinoma assessed with the monoclonal antibody MIB1 in paraffin sections. Laryngoscope 1997;107:531-6.

25. Seifert G, Donath K. Hybrid tumours of salivary glands: definition and classification of five rare cases. Eur J Cancer B Oral Oncol 1996;32B:251-9.

26. Nagao T, Sugano I, Ishida Y, Asoh A, Munakata S, Yamazaki $\mathrm{K}$, et al. Hybrid carcinomas of the salivary glands: report of nine cases with a clinicopathologic, immunohistochemical, and p53 gene alteration analysis. Mod Pathol 2002;15:72433.

27. Lewis JE, McKinney BC, Weiland LH, Ferreiro JA, Olsen KD. Salivary duct carcinoma: clinicopathologic and immunohistochemical review of 26 cases. Cancer 1996;77:223-30.

28. Kamio N, Tanaka Y, Mukai M, Ikeda E, Kuramochi S, Fujii M, et al. A hybrid carcinoma: adenoid cystic carcinoma and salivary duct carcinoma of the salivary gland: an immunohistochemical study. Virchows Arch 1997;430:495-500.

29. Snyder ML, Paulino AF. Hybrid carcinoma of the salivary gland: salivary duct adenocarcinoma adenoid cystic carcinoma. Histopathology 1999;35:380-3.

30. Gallo O, Franchi A, Bianchi S, Boddi V, Giannelli E, Alajmo E. p53 oncoprotein expression in parotid gland carcinoma is associated with clinical outcome. Cancer 1995;75:2037-44.

31. Kamio N. Coexpression of p53 and c-erbB-2 proteins is associated with histological type, tumour stage, and cell proliferation in malignant salivary gland tumours. Virchows Arch 1996;428:75-83.

32. Yamamoto Y, Virmani AK, Wistuba II, McIntire D, Vuitch F, Albores-Saavedra J, et al. Loss of heterozygosity and microsatellite alterations in p53 and RB genes in adenoid cystic carcinoma of the salivary glands. Hum Pathol 1996;27:120410.

33. Rosa JC, Felix A, Fonseca I, Soares J. Immunoexpression of c-erbB-2 and p53 in benign and malignant salivary neoplasms with myoepithelial differentiation. J Clin Pathol 1997; 50:661-3.

34. Grote HJ, Schneider-Stock R, Neumann W, Roessner A. Mutation of p53 with loss of heterozygosity in the osteosarcomatous component of a dedifferentiated chondrosarcoma. Virchows Arch 2000;436:494-7. 